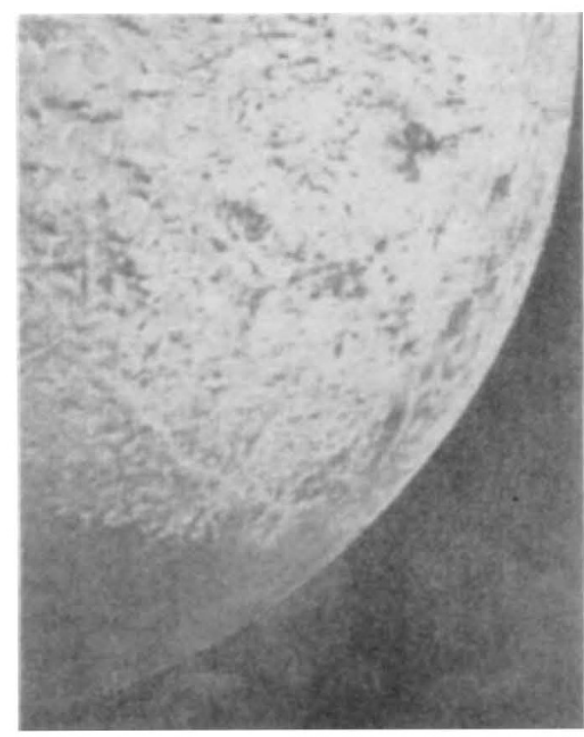

Triton: there's no such thing as a 'dead world'. unambiguously identified over several rotation periods. Around the Great Red Spot of Jupiter, for example, a multitude of recognizable smaller clouds pass by, permitting the rotation sense of the spot to be determined. But around Neptune's Great Dark Spot, the pattern of cirrus clouds is different on every image. Several days after the encounter, it was still impossible to decide if the Great Dark Spot is a cyclone or an anticyclone; as on Earth, the sense of rotation is different according to whether the spot is a low- or high-pressure region.

On some remarkable pictures, cirrus clouds are seen to cast shadows on the basal cloud layer; the height of the cirrus layer was estimated at about $50 \mathrm{~km}$. The dark spots appear to be about halfway between the high and low cloud layers.

The high clouds are methane condensations; the lower clouds are thought to consist of hydrogen sulphide. Sunlight acting on methane in the upper atmosphere probably creates heavier hydrocarbons, which freeze and sink; lower in the atmosphere, where the temperature rises again, they evaporate and decompose back to methane, which rises in plumes to form the visible clouds. On Neptune, further from the Sun than Uranus, most of the energy available to drive atmospheric dynamics is internal heat, welling up from below, rather than solar energy impinging on the upper atmosphere.

Passing beyond Neptune, Voyager 2 saved some of the most startling images of its career for last. Triton, a cold and presumably icy place $2,700 \mathrm{~km}$ in diameter, was far from dead. At a quick glance, cratering on Triton seems not to be heavy, implying that the surface has been processed somehow in fairly recent times the past 100 million years, for example. There had been suggestions that Triton would look 'new'; it has a uniquely odd orbit, circular but inclined to Neptune's axis, and retrograde. This makes it almost certainly a captured body, and suggests that in earlier times its orbit was probably not circular. As tidal forces circularized Triton's orbit, the strains on the moon's body would have heated it up, perhaps enough to melt water ice.

This indeed seems to have been the case. Parts of Triton's surface seem to have 'frozen lakes', resembling lunar maria. In places, the frozen lakes are stepped, suggesting a succession of meltings and re-freezings. But there is much more. Triton's inclined orbit, coupled with Neptune's, means that over a seasonal cycle of about a thousand years the Sun can be overhead anywhere from 50 degrees south to 50 degrees north. At the moment of Voyager's fly-by, the Sun was far to the south as seen from Triton, and near its south pole the surface markings were reminiscent of a martian summer, when the Sun is warm enough to evaporate the frozen carbon dioxide polar caps. On Triton, the ices are methane and nitrogen, but the process seems to be the same. Methane ice on Triton lends it a pinkish colour, darkly mottled by radiation damage from high-energy ionized particles which stream onto the the moon's surface and create darker hydrocarbons from the methane.

Elsewhere there is evidence of smaller flows that have filled in valleys and fissures. These are probably glaciers of methane and nitrogen which, like ice glaciers on Earth, can flow slowly over millions of years. Most bizarre of all are a few dark elongated streaks, tens of kilometres across. Initial suggestions that these might be wind-trails, formed of evaporating surface ices dispersed by the atmosphere, did not last long. Triton's atmosphere, inferred from the observation of a stellar occultation, has a pressure of no more than 10 millionths of Earth's, and is mostly nitrogen. There is too little of it to create a trail of sublimed ice.

The explanation settled on a few days after encounter was that the trails were from 'ice volcanoes'. Because nitrogen is just barely frozen at the surface temperature, an overburden of 20 or 30 metres is enough to cause nitrogen ice to liquefy. If fissures develop in the overlying ice, the liquid below will burst out, turn to gas which can shoot several kilometres above the surface, then condense and fall back. The thin surface layer so formed appears dark only in comparison with its surroundings, and may also entrap some of the sublimed dark ice particles in the vicinity.

A similar process may also account for yellow streaks seen on Io, one of Jupiter's moons, where underlying sulphur rather than nitrogen may rush up through surface cracks. Triton would then seem like a jigsaw of pieces from elsewhere in the Solar System, harbouring analogues of the martian polar caps, lunar maria and Io's vulcanism.

\section{Rings, arcs and moons}

BEFORE Voyager got close to Neptune, many astronomers were anticipating confirmation of the existence of many 'ring arcs', or partial rings. But by early this week, only four or five rings had been found, and all were complete, encircling the entire planet. Where did the arcs go?

Observations from Earth of occultations of stars passing behind Neptune suggested partial rings: stars were seen to flicker in brightness as they approached Neptune's disk, but no diminution in brightness was seen as the star emerged at the other side.

Altogether there was evidence for six ring arcs, at various distances from the planet. The early signs from Voyager looked good. JPL scientists announced three weeks before the encounter (see Nature $340,492 ; 1989)$ that they had detected faint reflected light from two partial rings.

But on Tuesday 22 August, new images revealed that the inner arc was in fact a complete ring, and that the other arc was longer than first thought. Much better pictures came down on Saturday, after closest approach, when Voyager could look back to see the ring system shining brightly in forward-scattered rather than reflected sunlight.

The full ring system revealed in these backlit images is complex. The two bright rings, 53,000 and $62,000 \mathrm{~km}$ in radius, are indeed both full rings, but the outer one has conspicuous clumps. Inside the inner bright ring is a faint and rather wide band, $42,000 \mathrm{~km}$ from Neptune and 2,000 km wide. Between the two bright rings is a fainter ring, but rather than a distinct ring this may be the edge of a tenuous, barely visible disk of material extending inwards to the inner bright ring, and possibly beyond.

The final assessment was that groundbased observers had indeed detected clumps in the outer ring - three of the observations gave about the right radius - and made the simple assumption that the ring was incomplete.

But one occultation measurement was both too far out and too strong to be consistent with any observed ring. Despite the astronomical odds, it seems that this occultation was caused by the then unknown moon $1989 \mathrm{~N} 2$, a $200-\mathrm{km}$-wide body happening, at a distance of 500 million $\mathbf{~ k m}$, to pass in front of a background star.

The final haul of moons was six, varying from $42,000 \mathrm{~km}$ out and $50 \mathrm{~km}$ in diameter to $120,000 \mathrm{~km}$ out and $500 \mathrm{~km}$ across. As with the other giant planets, the rings and moons form a complex system, whose properties will take years to puzzle out. Neptune's clumpy outer ring demands explanation, but none is immediately to hand: none of the moons is in the right place or has the right mass to be clearly responsible, by its gravitational influence, for the nonuniformities observed. 